\title{
Tunable few-electron double quantum dots and Klein tunnelling in ultra-clean carbon nanotubes
}

\author{
G. A. Steele ${ }^{1}$, G. Gotz ${ }^{1}, \&$ L. P. Kouwenhoven ${ }^{1}$ \\ ${ }^{1}$ Kavli Institute of NanoScience, Delft University of Technology, PO Box 5046, 2600 GA, Delft, The Nether- \\ lands.
}

Quantum dots defined in carbon nanotubes are a platform for both basic scientific studies[1, 2, $3,4,5]$ and research into new device applications[6]. In particular, they have unique properties that make them attractive for studying the coherent properties of single electron spins $[7,8,9$, $10,11]$. To perform such experiments it is necessary to confine a single electron in a quantum dot with highly tunable barriers[1], but disorder has until now prevented tunable nanotubebased quantum-dot devices from reaching the single-electron regime[2, 3, 4, 5]. Here, we use local gate voltages applied to an ultra-clean suspended nanotube to confine a single electron in both a single quantum dot and, for the first time, in a tunable double quantum dot. This tunability is limited by a novel type of tunnelling that is analogous to that in the Klein paradox of relativistic quantum mechanics.

Single spins in carbon nanotube quantum dots are expected to be very stable against both relaxation and decoherence[11]. Nuclear spins, the principal source of spin decoherence in GaAs[7, 8], can be completely eliminated and, furthermore, a strong spin-orbit interaction recently discovered in carbon nanotubes[9] enables all-electrical spin manipulation[10, 11], while preserving long spin relaxation and decoherence times[11]. Electron spins in carbon nanotube quantum dots are therefore attractive for implementation of a quantum bit (qubit) based on spin for applications in quantum-information processing[6]. In double quantum dot systems, precise control of the tunnel coupling between the two quantum dots, and between the quantum dots and the leads attached to them, is critically important for spin readout schemes[12, 13, 1], and also to prevent loss of spin and phase information through exchange of an electron with the leads.

Double quantum dots can also be used to explore novel quantum tunnelling phenomena. In Klein tunnelling $[14,15,16]$, for example, an electron tunnels with a high probability through a long and tall 
potential energy barrier when the height of the barrier is made comparable to twice the rest mass of the electron. It is not feasible to create such a barrier for free electrons due to the enormous electric fields required, but the low effective rest mass of the electrons in small bandgap nanotubes makes the observation of such Klein tunnelling in nanotube devices possible[16].

By depositing metallic gates isolated by a dielectric layer on top of a nanotube, several groups have demonstrated tunable double quantum dots in nanotubes lying on a substrate $[2,3,4,5]$. A disadvantage of this technique is that nanotubes in these devices suffer from significant disorder induced by the substrate and by the chemical processing required to fabricate the device. As the electron density is reduced, this random potential dominates and breaks the segment of nanotube into multiple disorder-induced "intrinsic" quantum dots before reaching the few-electron regime.

Wet etching of the device after fabrication to remove the substrate-induced disorder has been used previously to obtain single electron quantum dots in carbon nanotubes[17, 18], although experience has shown that the yield of such devices is quite low. Recently, a new fabrication method has been developed for producing ultra-clean quantum dots in suspended carbon nanotubes with a high yield in which all chemical processing is done before nanotube growth[19]. Studying single quantum dots in these devices has uncovered new carbon nanotube physics, including a strong spin orbit interaction due to the nanotube curvature[9] and evidence of Wigner crystallization of electrons at low density[20]. While devices fabricated in this way are extremely clean, they have some significant limitations: in particular, the confinement is produced only by Schottky barriers, which cannot be easily tuned in-situ. Furthermore, due to an insufficient number of local gates, it has not been possible to create a tunable double quantum dot in these ultra clean devices.

In order to overcome these limitations, we have developed a new method of integrating multiple local gates with the ultra clean fabrication. A schematic of the device is shown in Figure 1. As described in the Methods section, we grow a carbon nanotube over gates that are patterned in a thin doped silicon layer. Our current design provides three independent gates, although fabrication can easily be modified to include a scalable number of gates inside the trench (see Supplementary Information). In this letter, we use these three gates in two different ways. In device D1, with $L=1.5 \mu \mathrm{m}$, the gates are used to define a single electron and single hole quantum dot where electrons and holes are confined by tunable $p n$-junctions instead of Schottky 
contacts. In device D2, with $L=300 \mathrm{~nm}$, we rely on tunnel barriers from the Schottky contacts, but now use the three gates to create a tunable single electron and single hole double quantum dot.

In all previous measurements of quantum dots in carbon nanotubes containing a single electron, carriers were confined by Schottky barriers formed at the metal contacts $[9,17]$, or by potentials defined from trapped oxide charges[18]. In figure 2, we demonstrate a single electron quantum dot defined only by gate voltages. We begin by applying a negative voltage to the splitgates, creating a p-type nanotube source and drain on top of the oxide. Sweeping the backgate voltage $\mathrm{V}_{\mathrm{BG}}$, shown in figure $2 \mathrm{a}$, the current initially shows weak modulations from resonances in the leads when the suspended segment is p-type ( $p p p$ configuration), and is then completely suppressed as the suspended segment is depleted (pip configuration). As we sweep further, we form a pnp quantum dot showing clean Coulomb blockade, where single electrons in the suspended segment are confined by $p n$ junctions to the leads. Figure 2c shows a stability diagram as a function of both backgate and bias voltage, demonstrating that we have reached the single electron regime. As the confinement potential and doping profile are determined by our local gates, we can also confine single holes in an $n p n$ configuration in the same device simply by inverting the gate voltages, shown in figure 2 d. In figure $2 \mathrm{e}$ we show the current as a function of the backgate voltage and the voltage on the splitgates. In the left of the plot, the leads are doped p-type, and a positive backgate induces a single electron pnp quantum dot. In the right of the plot, the leads are doped n-type and a negative backgate induces a single hole npn quantum dot. By adjusting the splitgate voltages, the $p n$ junction width, and thus the tunnel barriers, can be tuned while keeping the electron number fixed (see figure $2 \mathrm{f}$ ).

In device D2, we use the gates in our design for a different purpose: here, we rely on less transparent Schottky contacts as incoming and outgoing tunneling barriers, and now use the backgate and the two splitgates as three independent local gates to create a double quantum dot potential in the nanotube with a tunable interdot coupling. Figure 3 shows the current through the device as a function of the two splitgate voltages. In the lower left and upper right regions of the plots, the two splitgates dope the two segments of the nanotube with carriers of opposite sign, resulting in a pn double quantum dot with an interdot barrier formed from a $p n$ junction. In the upper left (bottom right) corner, the two splitgates dope both sides of the nanotube p-type (n-type). In figure $3 \mathrm{a}, \mathrm{V}_{\mathrm{BG}}$ is set to ground, which gives a potential in the middle 
of the nanotube that is attractive for holes but repulsive for electrons. We consequently observe single dot behaviour for the first hole and weakly coupled double dot behaviour for the first electron. In figure 3b, we apply a positive backgate voltage, $\mathrm{V}_{\mathrm{BG}}=250 \mathrm{mV}$. The potential in the middle of the nanotube is now repulsive for holes: the first hole enters a weakly coupled double dot, while electrons fill a mostly single dot potential. (At some gate voltages, the presence of the oxide creates a non uniform potential which results in strongly coupled double dot instead of purely single dot behaviour. See section S1 of the Supplementary Information for further discussion.) By changing $\mathrm{V}_{\mathrm{BG}}$, we can continuously tune the interdot coupling in the few-electron and few-hole regime from weakly coupled double dot to single dot behaviour.

In figure 4, we investigate the tunable inderdot coupling in our double quantum dot more detail by studying current at the $(0,1 \mathrm{e}) \leftrightarrow(1 \mathrm{e}, 0)$ triple point transition of a weakly coupled double quantum dot. In a weakly coupled double quantum dot, current can only flow at specific values of the gate voltages, known as triple points, where the levels in the two dots are aligned, allowing an electron to tunnel from one dot to the other[21]. In figures $4 \mathrm{a}$ to $\mathrm{c}, \mathrm{V}_{\mathrm{BG}}$ is made more negative, creating a larger barrier for electron tunnelling between the dots, suppressing the current at the triple point. However, as we sweep $\mathrm{V}_{\mathrm{BG}}$ further, shown in figures $4 \mathrm{~d}$ and e, the current increases again, despite creating an even larger barrier for electron tunnelling.

The explanation of this curious increase of the current is a novel tunnelling process analogous to the tunnelling paradox in high energy physics proposed by Klein $[14,15,16]$. Specifically, we will define Klein tunnelling as any enhancement of the tunnelling of an electron through a barrier due the so-called negative energy solutions (positron states) that arise in relativistic quantum mechanics (see Supplementary Information for further discussion). In figure 4, the enhancement of the interdot coupling we observe at large tunnel barrier heights is an example of Klein tunnelling in a carbon nanotube, where now the valance band of the nanotube plays the role of the negative energy solutions in relativistic quantum mechanics. What is unique about the data in figure 4 is that we have created a direct implementation of Klein's gedanken experiment in our double quantum dot device, where we are able to tune continuously from the normal tunnelling regime to the Klein tunnelling regime simply by changing the barrier height with a gate voltage. We have also observed Klein tunnelling for holes (see Supplementary Information). In figure 4, what we observe is a kind of "virtual" Klein tunnelling, where the electron virtually occupies a state in the empty valance band in order 
to tunnel from the left to the right dot, similar to a cotunnelling process[22]. In addition to our observations in a double quantum dot, the $n p n$ data in figure 2 can be though of as a type of Klein tunnelling in a different regime, where the valance band is now occupied with holes, and where Klein tunnelling occurs by the electron sequentially tunneling across the two $p n$-junctions. This also emphasizes the close relation between Klein tunnelling in high energy physics and interband tunnelling phenomena in semiconductor physics, such as Zener tunnelling in insulators[23] and direct interband tunnelling in an Esaki diode[24].

Analyzing the current at the $(0,1 \mathrm{e}) \leftrightarrow(1 \mathrm{e}, 0)$ transition quantitatively using the result from Stoof and Nazarov $[25,26]$, we calculate the tunnel rates $\Gamma_{L}$ and $\Gamma_{R}$ of the barriers to the leads, and the interdot tunnel coupling $t_{c}$, shown in figure $4 \mathrm{~h}$. At these gate voltages, we are in the limit of weak interdot coupling: $t_{c} \sim 5$ $\mu \mathrm{V}<<\Gamma_{L}, \Gamma_{R} \sim 0.6 \mathrm{mV}$. The interdot coupling, $t_{c}$, is decreased from an initial value of $9 \mu \mathrm{V}$ to a minimum of $3 \mu \mathrm{V}$ as a function of backgate, before the onset of Klein tunnelling results in an increase up to $9 \mu \mathrm{V}$ as we approach gate voltages where an $n p n$ triple dot is formed. $\Gamma_{L}$ and $\Gamma_{R}$ are found to be independent of the backgate voltage, indicating that the backgate is not influencing the Schottky barrier transparency.

Finally, we comment that although we are in the appropriate double quantum dot coupling regime, we have not found evidence of spin blockade at any of the expected transitions[27]. (A parallel magnetic field of $1.5 \mathrm{~T}$ was applied to ensure that the nanotube valley degeneracy was lifted). One possible explanation for this is a singlet-triplet splitting in the $(0,2 \mathrm{e})$ state that is much smaller than the $3 \mathrm{mV}$ single particle spacing we observe in the single electron quantum dot. This could be an indication of Wigner crystal formation[20]: in a Wigner crystal, the electron wavefunction overlap is very small, and consequently the single-triplet splitting is strongly suppressed. This possibility will be investigated further using devices with more gates, which could allow us to probe the Wigner crystallization transition by tuning the quantum dot confinement potential.

We have presented a new technique for confining single electrons and single holes in ultra-clean carbon nanotubes. By eliminating disorder and incorporating local gates, a new level of control over single electron confinement has been achieved, allowing us to observe a novel type of tunnelling in a single electron carbon nanotube device. While our motivation for such a device comes from the spin physics of carbon nanotubes[28], the fabrication itself could have a much broader use in carbon nanotube applications, such as electrically doped $p n$ junctions for carbon nanotube optical emission[29], where low disorder and multiple gates for 
electrical control of $p n$ junctions could allow the development of new types of optically active devices.

\section{Methods}

Fabrication begins with a p++ Si wafer with $285 \mathrm{~nm}$ of thermal silicon oxide. On top of this, a $50 \mathrm{~nm}$ thick $\mathrm{n}++$ polysilicon gate layer is deposited, followed by a $200 \mathrm{~nm}$ LPCVD-TEOS oxide layer. Using electron-beam lithography and dry etching, a trench of approximately $300 \mathrm{~nm}$ deep is etched, forming the two splitgates from the $\mathrm{n}++\mathrm{Si}$ gate layer. A $5 / 25 \mathrm{~nm} \mathrm{~W} / \mathrm{Pt}$ layer is deposited to serve as source and drain contacts, and nanotubes are then grown from patterned Mo/Fe catalyst[30]. In about half of the devices, a single carbon nanotube is suspended across the trench making electrical contact to the source and drain. Transport through the devices is characterized at room temperature, and selected devices are cooled to $<300$ $\mathrm{mK}$ for low temperature transport measurements. In total, we have measured 11 devices at low temperatures, of which 4 reached the single electron regime. Here we present data from two small bandgap devices: D1 with $\mathrm{L}=1.5 \mu \mathrm{m}, \mathrm{W}=300 \mathrm{~nm}$ and bandgap $\mathrm{E}_{\mathrm{g}}=60 \mathrm{mV}$, and $\mathrm{D} 2$ with $\mathrm{L}=300 \mathrm{~nm}, \mathrm{~W}=500 \mathrm{~nm}$ and $\mathrm{E}_{\mathrm{g}}=$ $25 \mathrm{mV}$, where bandgaps are determined by subtracting the charging energy from the size of the empty dot Coulomb diamond.

\section{References}

[1] Hanson, R., Kouwenhoven, L. P., Petta, J. R., Tarucha, S. \& Vandersypen, L. M. K. Spins in few-electron quantum dots. Rev. Mod. Phys. 79, 1217-1266 (2007).

[2] Mason, N., Biercuk, M. J. \& Marcus, C. M. Local gate control of a carbon nanotube double quantum dot. Science 303, 655-658 (2004).

[3] Sapmaz, S., Meyer, C., Beliczynski, P., Jarillo-Herrero, P. \& Kouwenhoven, L. P. Excited state spectroscopy in carbon nanotube double quantum dots. Nano Lett. 6, 1350-1355 (2006).

[4] Jørgensen, H. I., Rasmussen, G. K., Hauptmann, J. R. \& Lindelof, P. E. Single wall carbon nanotube double quantum dot. Appl. Phys. Lett. 89, 232113 (2006). 
[5] Gräber, M. R. et al. Molecular states in carbon nanotube double quantum dots. Phys. Rev. B 74, $075427(2006)$.

[6] Loss, D. \& Divincenzo, D. P. Quantum computation with quantum dots. Phys. Rev. A 57, 120-126 (1998).

[7] Petta, J. R. et al. Coherent manipulation of coupled electron spins in semiconductor quantum dots. Science 309, 2180-2184 (2005).

[8] Koppens, F. H. L. et al. Universal phase shift and nonexponential decay of driven single-spin oscillations. Phys. Rev. Lett. 99, 106803 (2007).

[9] Kuemmeth, F., Ilani, S., Ralph, D. C. \& McEuen, P. L. Coupling of spin and orbital motion of electrons in carbon nanotubes. Nature 452, 448-452 (2008).

[10] Nowack, K. C., Koppens, F. H. L., Nazarov, Y. \& Vandersypen, L. M. K. Coherent control of a single electron spin with electric fields. Science 318, 1430-1433 (2007).

[11] Bulaev, D. V., Trauzettel, B. \& Loss, D. Spin-orbit interaction and anomalous spin relaxation in carbon nanotube quantum dots. Phys. Rev. B 77, 235301 (2008).

[12] Fujisawa, T., Austing, D. G., Tokura, Y., Hirayama, Y. \& Tarucha, S. Allowed and forbidden transitions in artificial hydrogen and helium atoms. Nature 419, 278-281 (2002).

[13] Elzerman, J. M. et al. Single-shot read-out of an individual electron spin in a quantum dot. Nature 430, 431-435 (2004).

[14] Klein, O. Die Reflexion von Elektronen an einem Potentialsprung nach der relativistischen Dynamik von Dirac. Z. Phys. 53, 157-165 (1929).

[15] Katsnelson, M. I., Novoselov, K. S. \& Geim, A. K. Chiral tunnelling and the Klein paradox in graphene. Nature Phys. 2, 620-625 (2006).

[16] Trauzettel, B., Bulaev, D. V., Loss, D. \& Burkard, G. Spin qubits in graphene quantum dots. Nature Phys. 3, 192-196 (2007). 
[17] Jarillo-Herrero, P., Sapmaz, S., Dekker, C., Kouwenhoven, L. P. \& van der Zant, H. S. Electron-hole symmetry in a semiconducting carbon nanotube quantum dot. Nature 429, 389-392 (2004).

[18] Minot, E. D., Yaish, Y., Sazonova, V. \& Mceuen, P. L. Determination of electron orbital magnetic moments in carbon nanotubes. Nature 428, 536-539 (2004).

[19] Cao, J., Wang, Q. \& Dai, H. Electron transport in very clean, as-grown suspended carbon nanotubes. Nature Mater. 4, 745-749 (2005).

[20] Deshpande, V. V. \& Bockrath, M. The one-dimensional Wigner crystal in carbon nanotubes. Nature Phys. 4, 314-318 (2008).

[21] van der Wiel, W. G. et al. Electron transport through double quantum dots. Rev. Mod. Phys. 75, 1+ (2002).

[22] De Franceschi, S. et al. Electron cotunneling in a semiconductor quantum dot. Physical Review Letters $\mathbf{8 6}, 878+(2001)$.

[23] Zener, C. A theory of the electrical breakdown of solid dielectrics. Roy. Soc. Proc. 145, 523-529 (1934).

[24] Esaki, L. New phenomenon in narrow germanium p-n junctions. Phys. Rev. 109, 603+ (1958).

[25] Stoof, T. H. \& Nazarov, Y. Time-dependent resonant tunneling via two discrete states. Phys. Rev. B 53, 1050-1053 (1996).

[26] Fujisawa, T. et al. Spontaneous emission spectrum in double quantum dot devices. Science 282, 932-935 (1998).

[27] Ono, K., Austing, D. G., Tokura, Y. \& Tarucha, S. Current rectification by Pauli exclusion in a weakly coupled double quantum dot system. Science 297, 1313-1317 (2002).

[28] Churchill, H. O. H. et al. Electron-nuclear interaction in ${ }^{13} \mathrm{C}$ nanotube double quantum dots. Preprint at http://arxiv.org/abs/0811.3236 .

[29] Misewich, J. A. et al. Electrically induced optical emission from a carbon nanotube FET. Science 300, 783-786 (2003). 
[30] Kong, J., Soh, H. T., Cassell, A. M., Quate, C. F. \& Dai, H. Synthesis of individual single-walled carbon nanotubes on patterned silicon wafers. Nature 395, 878-881 (1998).

\section{Acknowledgments}

It is a pleasure to acknowledge P. L. McEuen for the suggestion of using $p n$ junctions as tunable barriers, as well as D. Loss, T. Balder, I. T. Vink, R. N. Schouten, L. M. K. Vandersypen, and M. H. M. van Weert for useful discussions and suggestions. Supported by the Dutch Organization for Fundamental Research on Matter (FOM), the Netherlands Organization for Scientific Research (NWO), and the Japan Science and Technology Agency International Cooperative Research Project (JST-ICORP).

\section{Additional Information}

Supplementary information accompanies this paper at www.nature.com/naturenanotechnology. Reprints and permission information is available online at http://npg.nature.com/reprintsandpermissions/. Correspondence and requests for materials should be addressed to G.A.S.

The authors declare that they have no competing financial interests. 

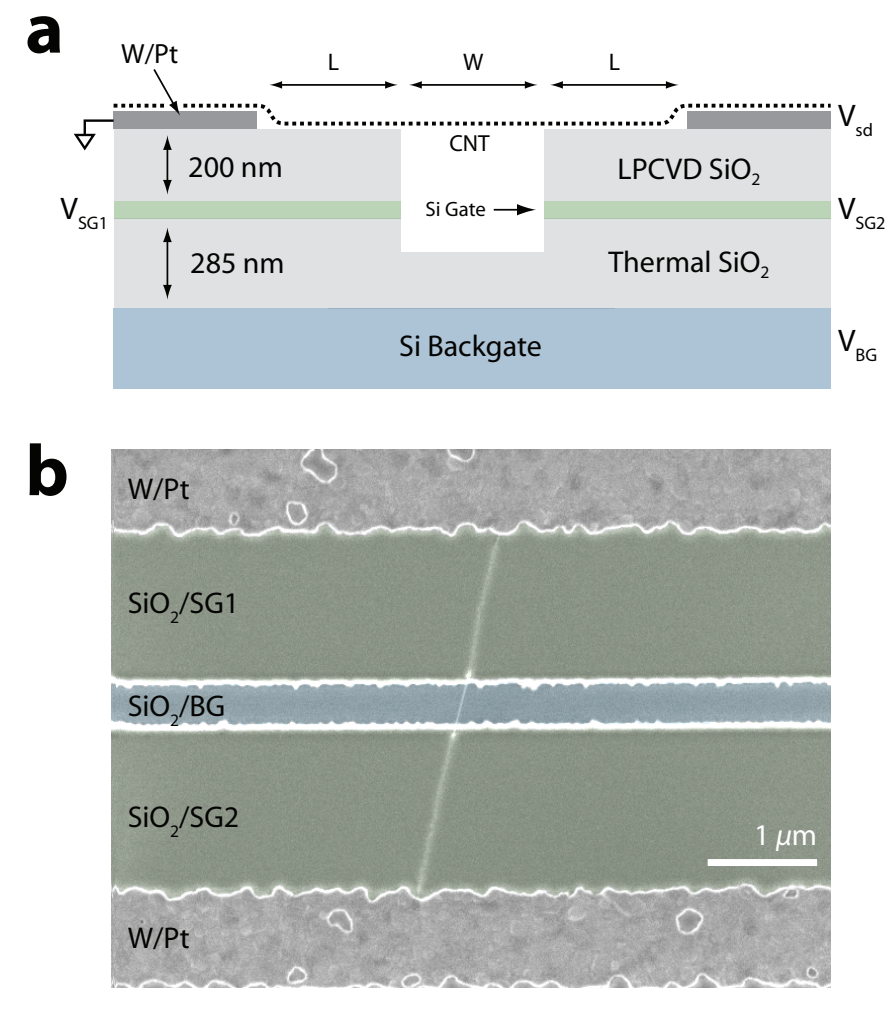

Figure S1: Integrating local gates with ultra-clean carbon nanotubes. a, A schematic of the device. A predefined trench is etched to create two splitgates from a $50 \mathrm{~nm}$ thick $\mathrm{n}++$ polysilicon gate layer between two silicon oxide layers. A Pt metal layer is deposited to act at as source and drain contacts, and a nanotube is then grown from patterned catalyst. Device $\mathrm{D} 1$ has $\mathrm{L}=1.5 \mu \mathrm{m}, \mathrm{W}=300 \mathrm{~nm}$, and $\mathrm{D} 2$ has $\mathrm{L}=300 \mathrm{~nm}, \mathrm{~W}=$ $500 \mathrm{~nm}$. b, In a subset of devices, a single nanotube bridges the trench, contacting the metal source and drain electrodes, as shown in this colourised SEM micrograph. The micrograph shows an example of a device with the same dimensions as device D1. 


\section{a}
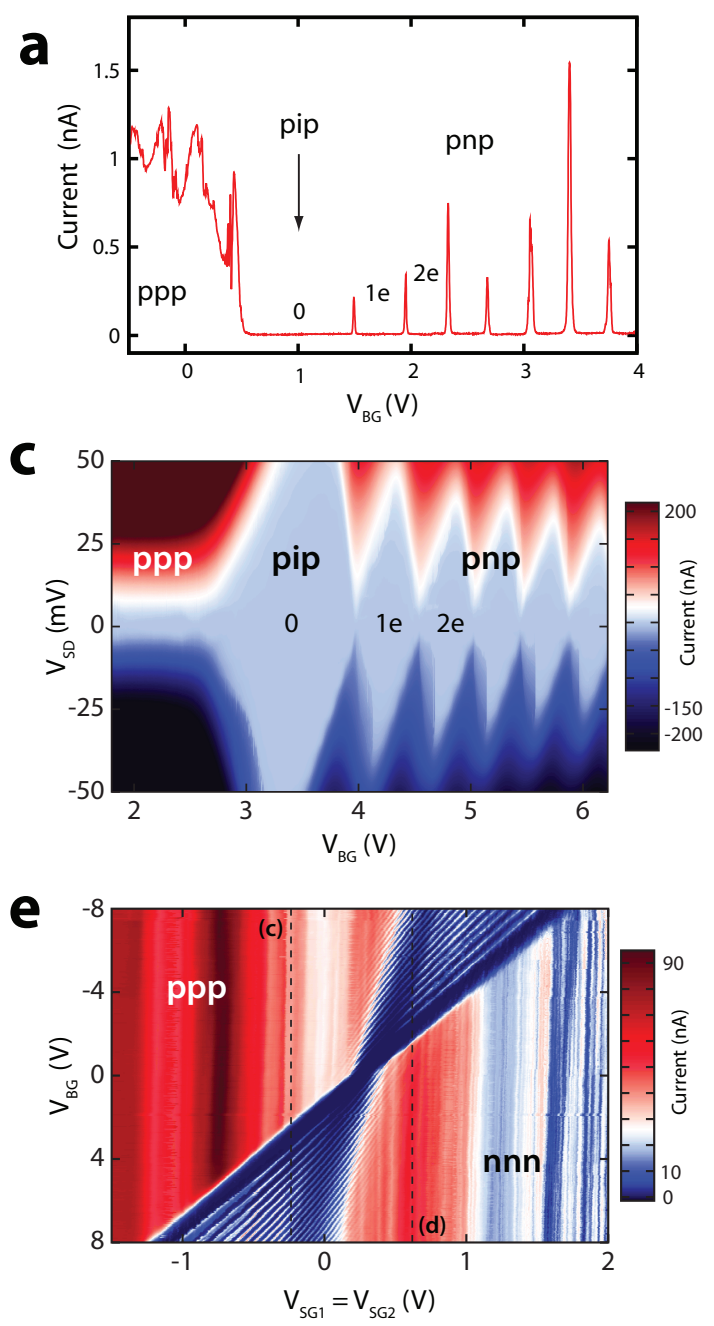

b
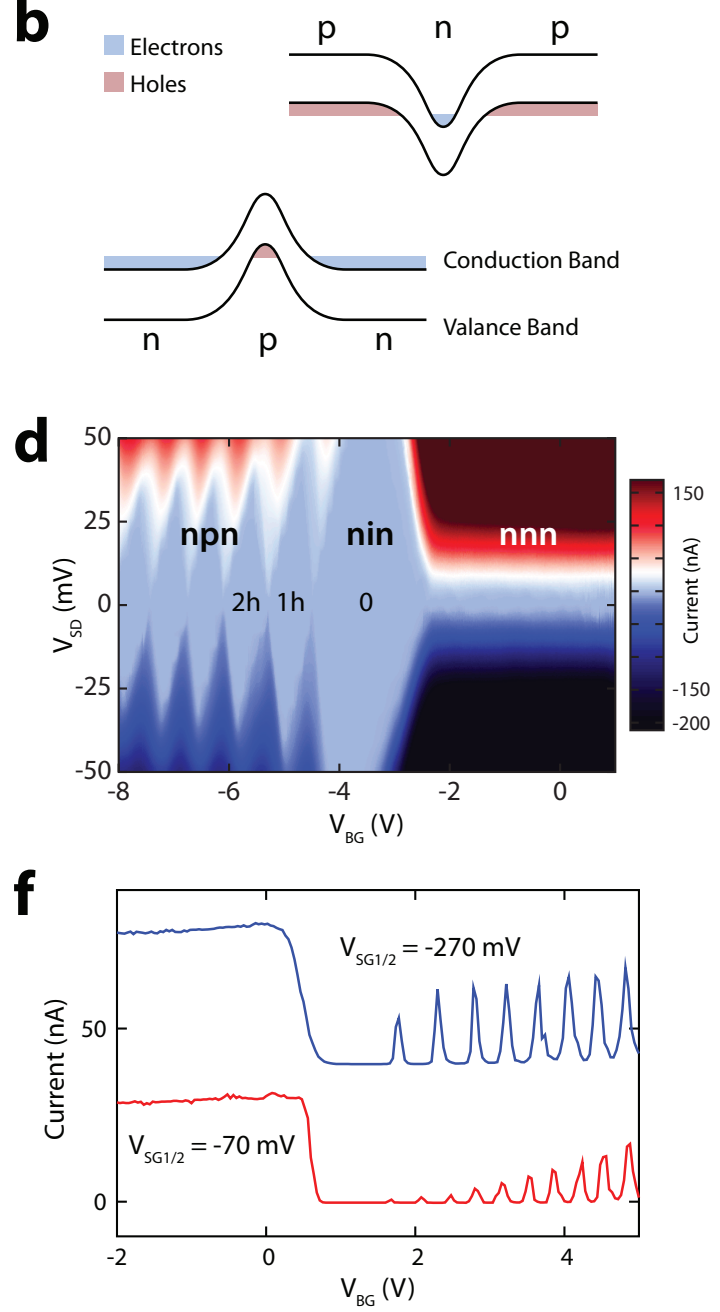

Figure S2: Gate defined single-electron and single-hole quantum dots. a, Coulomb peaks of a pnp quantum dot in device $\mathrm{D} 1$ taken at a $\mathrm{V}_{\mathrm{SG} 1}=\mathrm{V}_{\mathrm{SG} 2}=-50 \mathrm{mV}$ and $\mathrm{V}_{\mathrm{sd}}=1 \mathrm{mV}$. The splitgates are used to dope the NT source and drain leads with holes. As $V_{B G}$ is swept from negative to positive voltages, the suspended segment is depleted giving a pip configuration, followed by a pnp configuration as single electrons are filled in an n-type quantum dot, as illustrated in the energy diagrams in b. c, Stability diagram of the pnp dot: the charging energy of the first electron $\mathrm{E}_{\mathrm{c}}^{1 \mathrm{e}} \sim 40 \mathrm{meV}$ is remarkably large due to the weak capacitive coupling of the suspended segment to the gates and the metal source drain layers. d, The potential landscape in the device can be completely controlled by the gate voltages: by reversing the gate voltages, single holes are confined in a npn configuration. e, A 2D plot showing backgate sweeps at different splitgate voltages and $\mathrm{V}_{\mathrm{SD}}=10 \mathrm{mV}$. The two splitgates are set to the same voltage. The stability diagrams in $\mathbf{c}$ and $\mathbf{d}$ are taken at $\mathrm{V}_{\mathrm{SG} 1 / 2}$ values indicated by the arrows. (Resonances from residual disorder in the long NT leads can be seen as oscillations as a function of $\mathrm{V}_{\mathrm{SG} 1 / 2}$ in the ppp and $n n n$ configurations.) f, Using the splitgates, we can tune the width of the pn junction depletion region, and hence the tunnel barriers: at $\mathrm{V}_{\mathrm{SG} 1}=\mathrm{V}_{\mathrm{SG} 2}=-70 \mathrm{mV}$, the potential from the splitgates is shallow, giving a wide depletion region and a current of $0.5 \mathrm{nA}$ for the first electron Coulomb peak at $\mathrm{V}_{\mathrm{SD}}=10 \mathrm{mV}$. At $\mathrm{V}_{\mathrm{SG} 1}=$ $V_{\mathrm{SG} 2}=-270 \mathrm{mV}$, the potential across the $p n$ junction is steeper, now giving a narrower depletion region and a current of $13 \mathrm{nA}$ for the first electron. (The $\mathrm{V}_{\mathrm{SG} 1 / 2}=-270 \mathrm{mV}$ trace has been offset in $\mathrm{V}_{\mathrm{BG}}$ and in current.) 

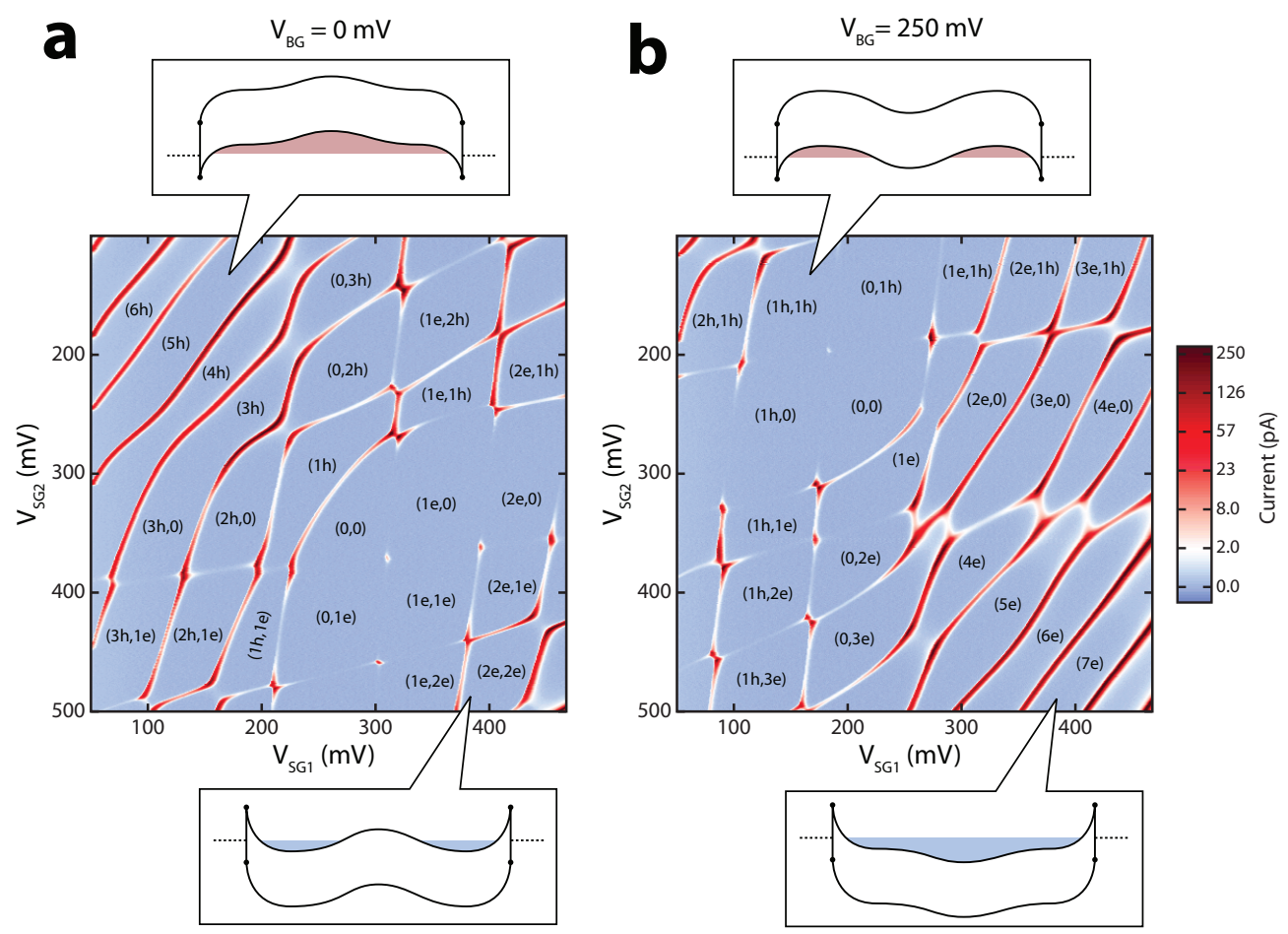

Figure S3: A tunable double quantum dot in the few-electron and few-hole regime. Current as a function of the two splitgate voltages at $\mathrm{V}_{\mathrm{SD}}=0.5 \mathrm{mV}$ for device $\mathrm{D} 2$. In device $\mathrm{D} 2$, electrons are confined in the nanotube by Schottky barriers at the metal contacts, with a potential that is tunable using the three gates. Electron and hole occupation numbers are determined from the transition to a $p n$ double quantum dot, as described in the Supplementary Information. $\mathbf{a}, \mathrm{V}_{\mathrm{BG}}=0$. At this voltage, a barrier for electrons is induced in the middle of the device. Electrons are added to a weakly coupled double dot potential, while holes are added to a single dot potential. b, $V_{\mathrm{BG}}=250 \mathrm{mV}$. A more positive $\mathrm{V}_{\mathrm{BG}}$ creates a double dot potential for holes and a single dot potential for electrons. The interdot coupling for both the electron and the hole double dot can be tuned continuously using the backgate voltage. 

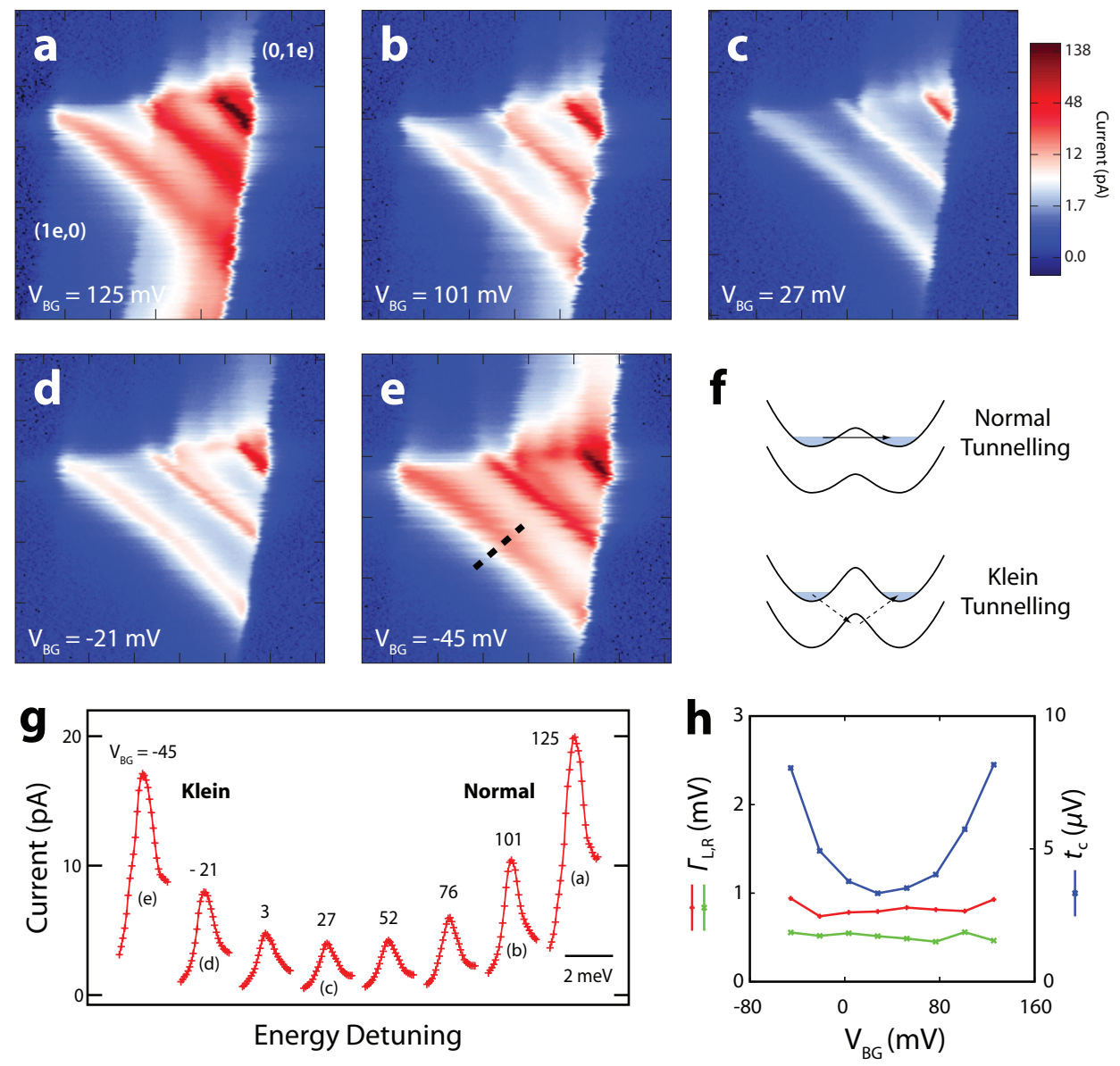

Figure S4: Klein tunnelling in a single electron double quantum dot. Current at the $(1 \mathrm{e}, 0) \leftrightarrow(0,1 \mathrm{e})$ triple point for a single electron double quantum dot at $\mathrm{V}_{\mathrm{SD}}=5 \mathrm{mV}$. (Note that the interdot capacitance $E_{\mathrm{c}}^{\text {inter }} \sim 0.2$ $\mathrm{mV}$ is much smaller than the bias, and thus the triple point bias triangles for the electron and hole cycle[21] strongly overlap.) Transitions to the excited state of the outgoing dot are visible as lines in the triangle running parallel to the baseline give a quantized level spacing of $3 \mathrm{mV}$, consistent with a dot length of $\sim 500 \mathrm{~nm}$. In a through $\mathbf{c}$, the backgate is made more negative, creating a larger barrier for electron tunnelling. As a result, the current through the double dot is decreased. In $\mathbf{d}$ and $\mathbf{e}$, however, the current begins to increase again despite a larger barrier for electron tunnelling. $\mathbf{f}$, This increase in current results from tunnelling of an electron below the barrier through a virtual state in the valence band, analogous to Klein tunnelling in high energy physics. g, Line cuts of the triple point data in a-e showing the current for the ground state baseline transition at different backgate voltages. The line cuts are taken along the dashed line in $\mathbf{e}$. The $\mathrm{x}$-axis shows the distance along this line converted into the energy detuning between the left and right dot ground state levels. For the rightmost traces, interdot tunnel coupling is mediated by normal electron tunnelling, while for the leftmost traces, Klein processes provide the interdot tunnel coupling. $\mathbf{h}$, Parameters from a fit to the Stoof-Nazarov equation. The interdot tunnel coupling initially decreases as the barrier height increases $\left(V_{B G}=125\right.$ to $\left.27 \mathrm{mV}\right)$, and then increases due to the onset of Klein tunnelling as the barrier height becomes comparable to the bandgap ( $\mathrm{V}_{\mathrm{BG}}=27$ to $-45 \mathrm{mV}$ ). 


\title{
Tunable few-electron double quantum dots and Klein tunnelling in ultra-clean carbon nanotubes: Supplementary Information
}

\author{
G. A. Steele ${ }^{1}$, G. Gotz ${ }^{1}, \&$ L. P. Kouwenhoven ${ }^{1}$ \\ ${ }^{1}$ Kavli Institute of NanoScience, Delft University of Technology, PO Box 5046, 2600 GA, Delft, The Nether- \\ lands.
}

\section{S1 Determining electron numbers}

Absolute electron numbers in the device are identified by the transition from a $n n$ or a $p p$ single dot to a $p n$ or $n p$ double dot, as shown in figure $\mathrm{S} 1$. For example, at $\mathrm{V}_{\mathrm{SG} 1}=-250 \mathrm{mV}$ and $\mathrm{V}_{\mathrm{SG} 2} \sim 260 \mathrm{mV}$, we remove the last hole from the right side of the nanotube, $(\mathrm{p}, \mathrm{p}) \rightarrow(\mathrm{p}, 0)$. As we sweep $\mathrm{V}_{\mathrm{SG} 2}$ further, at $\mathrm{V}_{\mathrm{SG} 2} \sim 380 \mathrm{mV}$, we fill an electron into the right dot. Here we see an abrupt transition from single dot behaviour to double dot behaviour, signaling the transition to a $(\mathrm{p}, \mathrm{n})$ double dot. This transition allows us to clearly identify the electron numbers in the device. The electron number assignment was also confirmed by large bias Coulomb diamond measurements such as those shown in figure 2 of the main text.

At $\mathrm{V}_{\mathrm{SG} 1} \sim 500 \mathrm{mV}$, the device suffers from a "switch" in gate voltage: this switch, which appeared on the third cooldown of the device, is likely due to a charge trap in the oxide. Aside from this, the device is extremely stable. It is also very robust with respect to thermal cycling: after 2 thermal cycles including exposure to air, the barrier transparencies were unchanged and the position of the first Coulomb peak moved by less than $50 \mathrm{mV}$ in gate space.

Note also that although the backgate voltage used in figure S1 should result in single dot behaviour for holes, the data show some bending of the Coulomb peak trajectories along the $(\mathrm{p}, \mathrm{p})$ to $(0, \mathrm{p})$ and $(\mathrm{p}, 0)$ transitions, indicating a strongly tunnel coupled double dot type of behaviour. It is also visible along the $(\mathrm{n}, \mathrm{n})$ to $(\mathrm{n}, 0)$ and $(0, \mathrm{n})$ transitions in figure $3(\mathrm{~b})$ of the main text, and at higher electron numbers in figure S1. This results from a somewhat non uniform potential induced by the presence of the oxide under part of the tube, likely due to a combination of trapped charges in the oxide and the abrupt change in dielectric constant. 


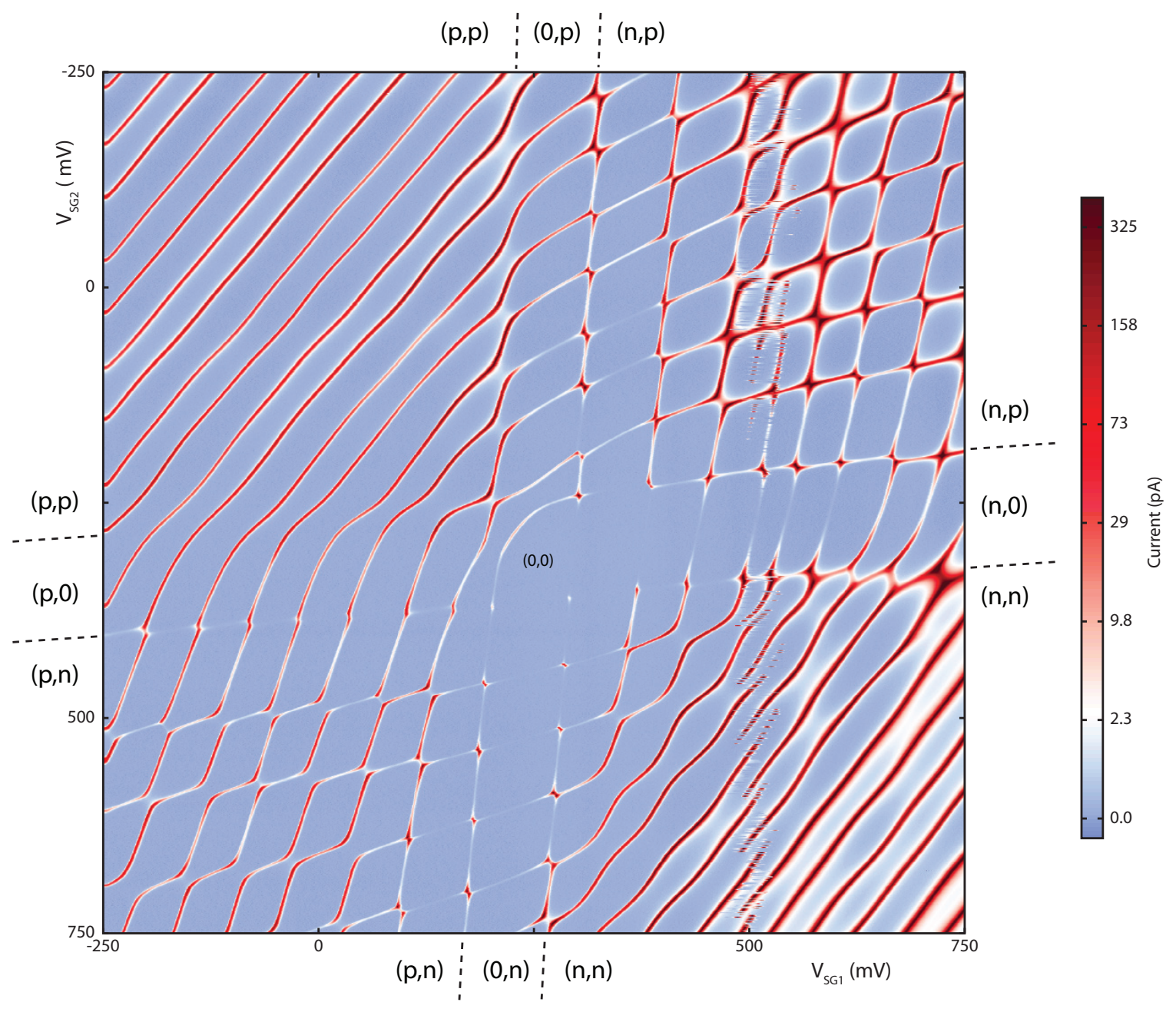

Figure S1: A 2D splitgate sweep over a larger range used to determine electron numbers from the transition to a pn double quantum dot. Data is taken at $\mathrm{V}_{\mathrm{BG}}=50 \mathrm{mV}$ and $\mathrm{V}_{\mathrm{SD}}=0.5 \mathrm{mV}$. 

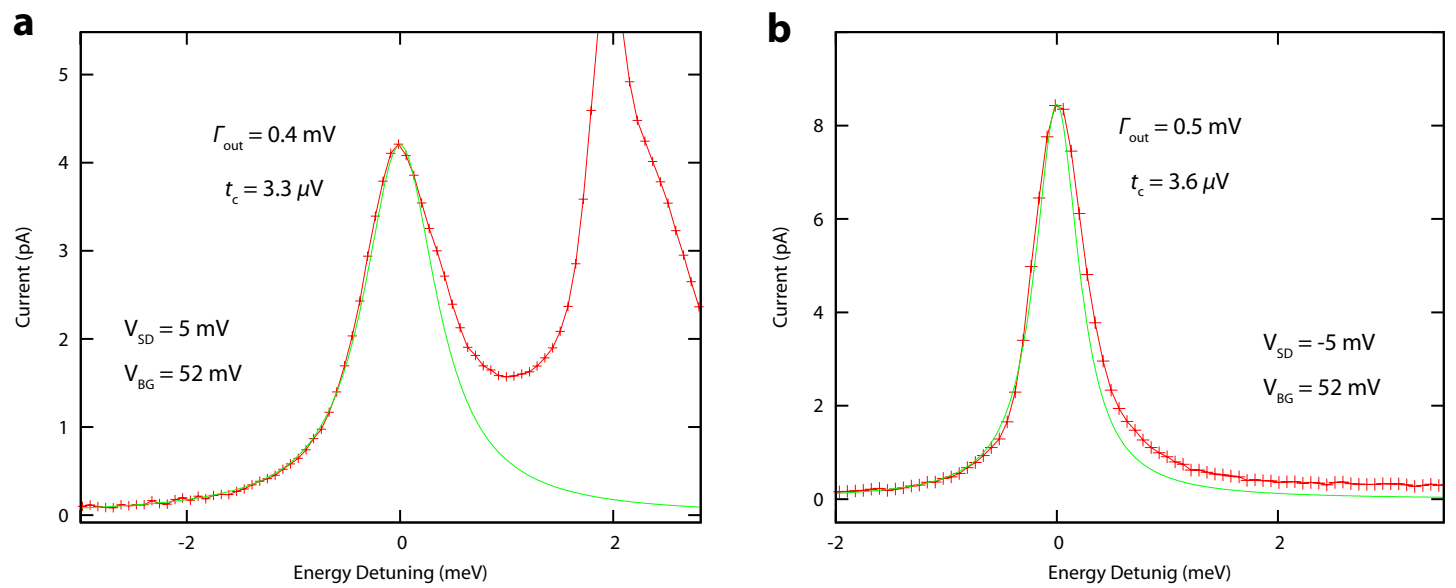

Figure S2: Fit of $(0,1 \mathrm{e}) \leftrightarrow(1 \mathrm{e}, 0)$ transition at $\mathrm{V}_{\mathrm{BG}}=52 \mathrm{mV}$ to the Stoof-Nazarov theoretical result for a, positive and $\mathbf{b}$, negative bias. A detuning independent inelastic contribution to the current of $350 \mathrm{fA}$ is clearly visible in the reverse bias trace. This inelastic current is also present in $\mathbf{a}$, but is more difficult to identify due to a nearby excited state of the outgoing dot in forward bias.

\section{S2 Stoof-Nazarov Equation}

To analyze the data quantitatively, we fit the current at the ground state to ground state transition along the baseline of the triple point bias triangle as a function of energy detuning $\epsilon$ to the expression from Stoof and Nazarov[1, 2]. By performing such an analysis, we are able to isolate the contribution of the middle tunnel barrier from the measurement of the current through the double quantum dot. For a interdot tunnel coupling $t_{c}$ and tunnel rates $\Gamma_{L, R}$ to the left and right leads, the elastic current in a double quantum dot is given by:

$$
I_{e l}(\epsilon)=\frac{e t_{c}^{2} \Gamma_{R}}{t_{c}^{2}\left(2+\Gamma_{R} / \Gamma_{L}\right)+\Gamma_{R}^{2} / 4+(\epsilon / h)^{2}}
$$

In the limit of weak interdot tunnel coupling, $t_{c}<<\Gamma_{L}, \Gamma_{R}$, this reduces to a simple Lorentzian line shape of the form:

$$
I_{e l}(\epsilon)=\frac{4 e t_{c}^{2} / \Gamma_{R}}{1+\left(2 \epsilon / \Gamma_{R} h\right)^{2}}
$$

A fit of the data to equation 2 for a single electron double dot is shown in figure S2. The fit was performed for $\epsilon<0$ to isolate the purely elastic contribution to the current. For $\epsilon>0$, the fit deviates from the Lorentzian 
lineshape due to inelastic processes[3].

\section{S3 Relativistic tunnelling through a barrier and the Klein Paradox}

Consider an electron of energy $E$ and momentum $\hbar k$ incident on a square barrier of height $V$ as shown in figure S3. We are interested in the probability that the electron is transmitted to $x>L$ using the Dirac equation. The solutions of the Dirac equation have two branches[4]: a set of positive energy solutions with $E>0$ and a set of negative energy solutions with $E<0$. The two branches are separated by an energy gap $2 m c^{2}$. The vacuum state is interpreted as having the negative energy solutions filled with electrons (the "Dirac sea"), and a hole in the Dirac sea is then interpreted as a positron. For a barrier height that is small compared to $2 m c^{2}$, shown in figure S3(a), the Dirac equation gives a wavefunction that decays exponentially inside the barrier: for an incident energy $E \ll V$, the probability of the electron tunnelling to the region $x>L$ is small. This is also what is predicted by the non-relativistic Schroedinger equation.

However, if the barrier height becomes very large, so that $V$ is comparable to $2 m c^{2}$, the negative energy solutions of the Dirac equation strongly modify the tunnelling process. In particular, Klein noticed that for $V \sim 2 m c^{2}$, as shown in S3(b) and S3(c), an electron moving at non-relativistic speeds incident on the barrier at position $x=0$ can tunnel to $x>L$ on the other side of the barrier with nearly unity probability. In the context of the non-relativistic Schroedinger equation, such a high tunnelling probability would be completely unexpected, hence the idea of such tunnelling as a paradox.

The tunnelling enhancement can be divided in to two regimes, illustrated in figures S3(b) and S3(c).

We will refer to the first, illustrated in figure S3(b), as the (Klein) Tunnelling regime[5]. Here, the electron propagates inside the barrier as an evanescent wave, but the transmission probability can be very high since the decay length is significantly longer that that from the Schroedinger equation due to the negative energy solutions. We will refer to the second regime, shown in figure S3(c), as the (propagating) Klein regime. Here, the wavefunction in the barrier is oscillatory in nature and does not decay. Both cases are examples of what we will call non-classical "Klein Tunnelling" in which the electron emerges at $x>L$ with a much higher probability than that predicted by the Schroedinger equation.

The electronic spectrum of a carbon nanotube at low energies is also given by a Dirac equation that 
a

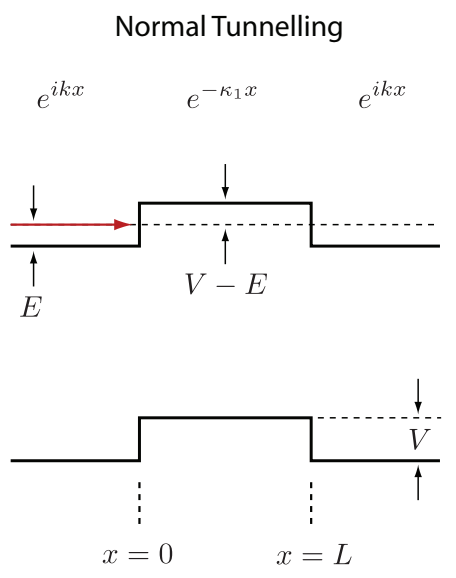

b

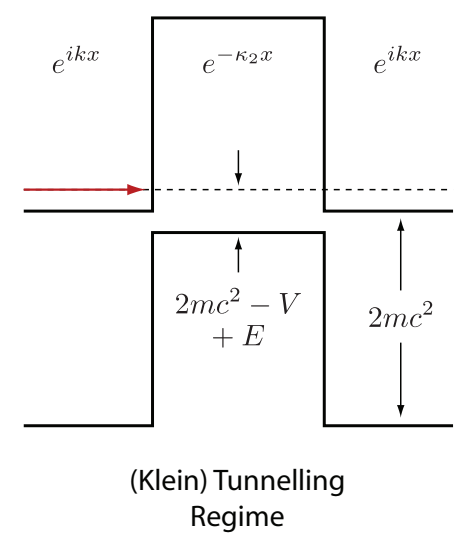

C

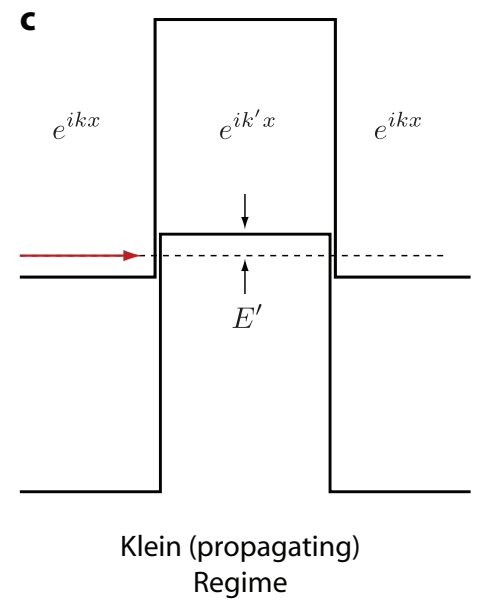

Figure S3: Relativistic tunnelling through a barrier. Positive energy solutions of the Dirac equation are separated from the negative energy solutions by a an energy gap $2 m c^{2}$. We consider the probability that an electron incident on a barrier of height $V$ at $x=0$ with energy $E$ is transmitted to the region $x>L$. a, For $V \ll 2 m c^{2}$, the wavefunction inside the barrier decays exponentially with a decay length $\kappa_{1}=\sqrt{2 m(V-E) / \hbar}$, as predicted by the non-relativistic Schroedinger equation. We refer to this as the "Normal" tunnelling regime. b, For $V$ slightly less than $2 m c^{2}$, the wavefunction also decays exponentially inside the barrier. However, due to the nearby negative energy solutions of the Dirac equation, the decay length is now much longer, given by $\kappa_{2}=$ $\sqrt{2 m\left(2 m c^{2}-V+E\right) / \hbar}$, and the transmission probability is much higher than that predicted by the Schroedinger equation. We refer to this as the (Klein) Tunnelling regime. c, For $V>2 m c^{2}$, the electron now propagates inside the barrier without decaying by occupying a negative energy solution of the Dirac equation. Inside the barrier, the wavefunction is a plane wave $e^{i k^{\prime} x}$ with energy $E^{\prime}=V-2 m c^{2}-E$. We refer to this as the Klein (propagating) regime. 
is the same as that for normal electrons[6, 7], but with $2 m c^{2}$ replaced by the bandgap $E g$, and the speed of light $c$ replaced by the Fermi velocity of graphene $v_{F}\left(\sim 0.9 \times 10^{6} \mathrm{~m} / \mathrm{s}\right)$. Free electrons in the Dirac equation correspond to electrons in the conduction band of the nanotube, and positrons in the Dirac equation correspond to holes in the valance band. Thus, it should be possible to observe phenomena analogous to the two Klein tunnelling regimes of S3(b) and S3(c) in a carbon nanotube device.

In figure 2 of the main text, we present data demonstrating a single hole $n p n$ and single electron $p n p$ quantum dot. The current that we observe at the Coulomb peaks can be considered as an example of the (propagating) Klein regime illustrated in figure S3(c), where the potential barrier from our gate voltages is larger than the bandgap. Figure 2(d) from the pnp configuration corresponds to the (propagating) Klein regime for positrons, and figure 2(c) from the $n p n$ configuration corresponds to the same regime for electrons.

In figure 4 of the main text, we show an example of the (Klein) tunnelling regime of figure S3(b). In the data, we observe a continuous transition from the normal tunnelling regime to that where the negative energy solutions of the Dirac equation provide an enhancement of electron tunnelling, as in the original Klein gedanken experiment. We also note that the unusual tunnelling process shown in figure S3(b), where the decay length in the barrier is increased due to the negative energy states, has recently been proposed as a mechanism of generating exchange coupling between two distant quantum dots in graphene nanoribbons[7]. Our experiment demonstrates explicitly such tunnelling in a carbon nanotube.

\section{S4 Klein tunnelling for a single hole double quantum dot}

In figure S4(a), we show Klein tunnelling for a single hole double quantum dot. Qualitatively, the process is the same as that for the single electron double dot. In the single hole double dot, the tunnel rates to the leads are smaller by a factor of 2-3 compared to the single electron double dot: in the device, the Fermi level pinning at the metal contacts is such that electrons see a smaller Schottky barrier. This can also be seen in figure S1, where the electron single dot peaks show more current and broadening than those of the hole single dot. 
a
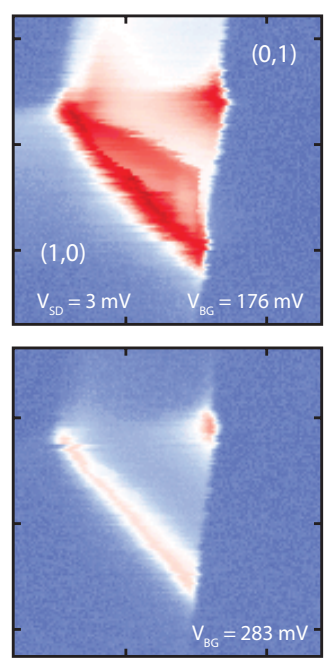
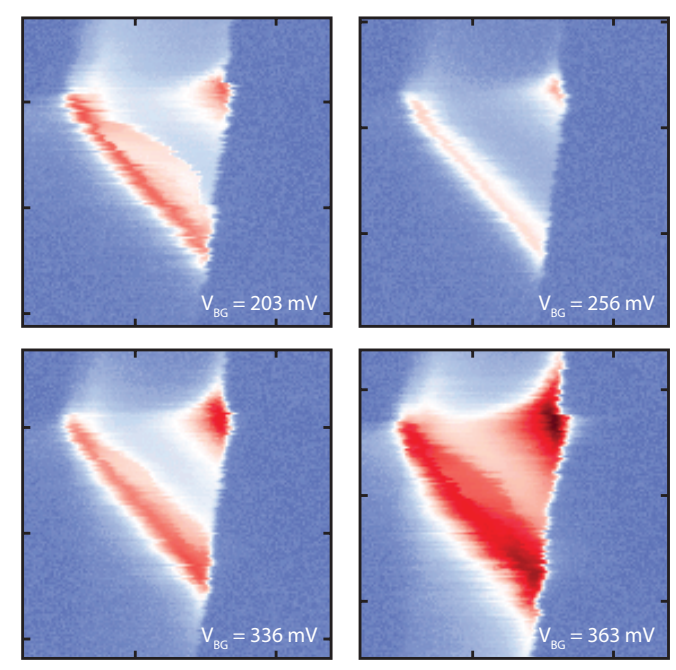

b

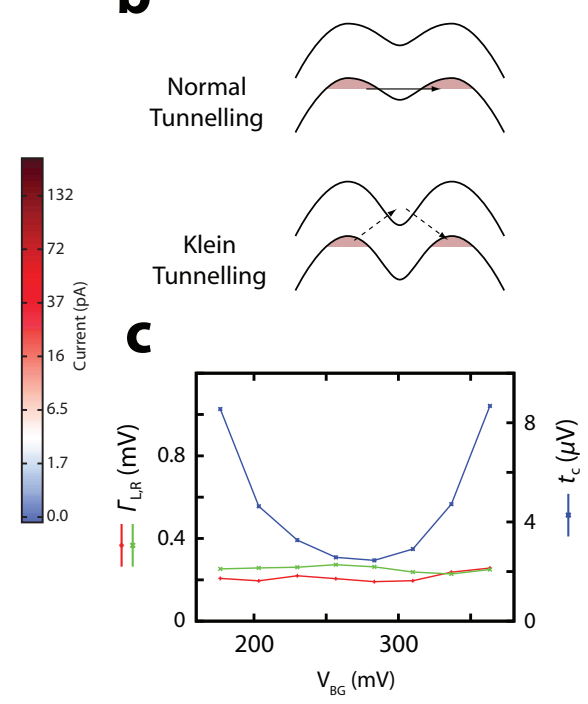

Figure S4: a,b Klein tunnelling at the $(0,1 \mathrm{~h}) \leftrightarrow(1 \mathrm{~h}, 0)$ single hole double dot transition and $\mathbf{c}$, parameters from a fit to the Stoof-Nazarov expression.

\section{S5 Extending fabrication to include more gates}

In the device studied in this paper, the incoming and outgoing barriers of the double dot were formed by Schottky barriers at the metal contacts. Because we have only three gates, for the double dot we could not tune the incoming and outgoing barriers independently of the electron number. As a result, we have significant lifetime broadening of the energy levels in the double dot configuration.

To overcome this, we can easily extend the fabrication to include more gates inside the trench, as shown schematically in figure S5(a). As an example of this, a test structure using this type of fabrication is shown in figure $\mathrm{S} 5(\mathrm{~b})$.

In addition to tuning $\Gamma_{L, R}$ independent of electron number, these gates could also be used to tune the single dot confinement potential. If Wigner crystallization is indeed responsible for the lack of spin blockade in our current device, we should be able to observe a transition to a finite singlet-triplet splitting by tuning the confinement energy using these extra gates. 

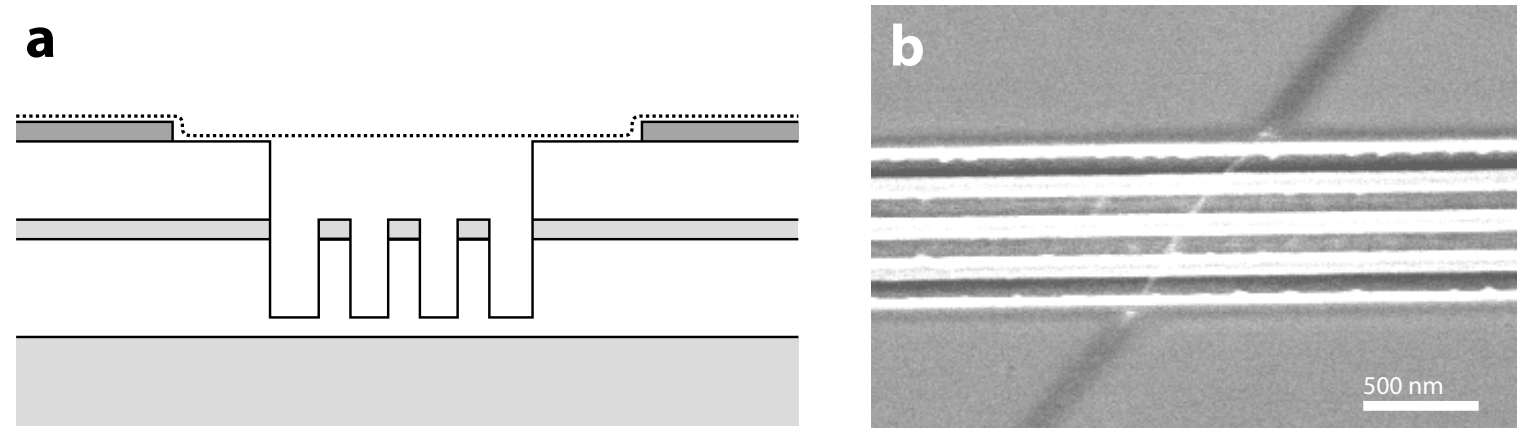

Figure S5: a, Schematic of device with more gates in the trench and $\mathbf{b}$, an SEM micrograph of a test device with a suspended nanotube.

\section{References}

[1] Stoof, T. H. \& Nazarov, Y. Time-dependent resonant tunneling via two discrete states. Phys. Rev. B 53, 1050-1053 (1996).

[2] Fujisawa, T., van der Wiel, W. G. \& Kouwenhoven, L. P. Inelastic tunneling in a double quantum dot coupled to a bosonic environment. Physica E 7, 413-419 (2000).

[3] Fujisawa, T. et al. Spontaneous emission spectrum in double quantum dot devices. Science 282, 932-935 (1998).

[4] See, for example, Perkins, D. H., Introduction to High Energy Physics (Addison-Wesley, Menlo Park, 1987).

[5] Bernardini, A. E. Relativistic tunneling and accelerated transmission. J. Phys. A 41, 215302+ (2008).

[6] Bulaev, D. V., Trauzettel, B. \& Loss, D. Spin-orbit interaction and anomalous spin relaxation in carbon nanotube quantum dots. Phys. Rev. B 77, 235301 (2008).

[7] Trauzettel, B., Bulaev, D. V., Loss, D. \& Burkard, G. Spin qubits in graphene quantum dots. Nature Phys. 3, 192-196 (2007). 